\title{
Tunable Subluminal Propagation of Narrow-band X-Ray Pulses
}

\author{
Kilian P. Heeg, ${ }^{1}$ Johann Haber, ${ }^{2}$ Daniel Schumacher, ${ }^{2}$ Lars Bocklage, ${ }^{2}$ Hans-Christian Wille, ${ }^{2}$ Kai S. Schulze, ${ }^{3,4}$ \\ Robert Loetzsch, ${ }^{3,4}$ Ingo Uschmann, ${ }^{3,4}$ Gerhard G. Paulus, ${ }^{3,4}$ Rudolf Rüffer, ${ }^{5}$ Ralf Röhlsberger, ${ }^{2}$ and Jörg Evers ${ }^{1}$ \\ ${ }^{1}$ Max-Planck-Institut für Kernphysik, Saupfercheckweg 1, 69117 Heidelberg, Germany \\ ${ }^{2}$ Deutsches Elektronen-Synchrotron DESY, Notkestraße 85, 22607 Hamburg, Germany \\ ${ }^{3}$ Institut für Optik und Quantenelektronik, Friedrich-Schiller-Universität Jena, Max-Wien-Platz 1, 07743 Jena, Germany \\ ${ }^{4}$ Helmholtz-Institut Jena, Fröbelstieg 3, 07743 Jena, Germany \\ ${ }^{5}$ ESRF-The European Synchrotron, CS40220, 38043 Grenoble Cedex 9, France
}

(Received 29 August 2014; published 18 May 2015)

\begin{abstract}
Group velocity control is demonstrated for x-ray photons of $14.4 \mathrm{keV}$ energy via a direct measurement of the temporal delay imposed on spectrally narrow x-ray pulses. Subluminal light propagation is achieved by inducing a steep positive linear dispersion in the optical response of ${ }^{57} \mathrm{Fe}$ Mössbauer nuclei embedded in a thin film planar x-ray cavity. The direct detection of the temporal pulse delay is enabled by generating frequency-tunable spectrally narrow $\mathrm{x}$-ray pulses from broadband pulsed synchrotron radiation. Our theoretical model is in good agreement with the experimental data.
\end{abstract}

Strong nonlinear interaction of light with matter is a key requirement for fundamental and applied quantum optical technologies alike. Since conventional materials typically exhibit weak nonlinearities, the ultimate quest for strong nonlinear interactions of individual quanta has led to the development of a number of methods to significantly enhance nonlinear light-matter interactions. Among the most prominent ones are coherently prepared media based on electromagnetically induced transparency, subluminal light, and related effects [1,2], as well as cavity-enhanced light matter interactions [3].

Recently, nuclear quantum optics featuring the interaction of x-ray light with Mössbauer nuclei in the few keV transition energy range has gained considerable momentum, both theoretically [4-9] and experimentally [10-20]. Interestingly, these experiments operate with less than one resonant $\mathrm{x}$-ray photon per pulse on average due to restrictions in the available $x$-ray light sources. This raises the question of whether coherent or cavity-based enhancement techniques could be utilized to realize nonlinear lightmatter interactions in nuclear quantum optics despite the low number of resonant photons.

Here, we report on a first step towards this goal, and demonstrate group velocity control of spectrally narrow $\mathrm{x}$-ray pulses (SNXP). Subluminal light propagation is achieved by inducing a steep positive linear material dispersion, and verified by direct measurements of the temporal delay imposed on the SNXP. For this purpose, we manipulate the optical response of the $\omega_{0}=14.4 \mathrm{keV}$ Mössbauer resonance (single nucleus linewidth $\gamma=$ $4.7 \mathrm{neV}$ ) of a large ensemble of ${ }^{57} \mathrm{Fe}$ nuclei embedded in a thin film planar $\mathrm{x}$-ray cavity. Our approach thereby combines coherent control, as well as cooperative and cavity enhancements of light-matter interaction in a single setup. To enable the direct detection of the temporal pulse delay, we further propose and implement a flexible scheme to generate frequency-tunable SNXP from broadband synchrotron radiation for applications in $\mathrm{x}$-ray quantum optics. Our theoretical model is in good agreement with the experimental data.

Subluminal light was first demonstrated in the visible frequency range [21-23], and by now has been implemented in a number of platforms [2], particularly also in cavity settings [24,25]. Manipulation of light propagation has also been reported in the x-ray regime. In Ref. [12], a delayed peak in the transmitted $\mathrm{x}$-ray light intensity has been observed. In this case, however, the pulse delay is induced by the propagation of the light through a doublet absorber structure rather than electromagnetically induced transparency or related effects, and can be interpreted as arising from transitions between super- and subradiant states. Also coherent storage of light via rapid control of the applied quantization field has been achieved [10]. Other experiments with nuclei observed electromagnetically induced transparency [15], related spontaneously generated coherences with equivalent susceptibilites [16], or other transparency mechanisms [11,26]. However, these experiments did not study the delay or the actual pulse propagation. In contrast, in our experiment, we induce slow light via a steep linear dispersion, and verify the $\mathrm{x}$-ray group velocity control via a direct observation of the temporal pulse delay.

Group velocity control.-From studies in atomic media it is known that the group velocity and hence the time delay is related to the frequency dependence of the medium susceptibility $[1,27]$. During its propagation, a spectrally narrow pulse accumulates a phase $\exp (i k L)$, where $L$ is the propagation length, $k=2 \pi n / \lambda_{0}$ is the wave vector in the 
medium and $n \approx 1+\chi / 2$ the refractive index. A steep linear dispersion directly influences the group velocity $v_{g}=\partial \omega / \partial k$ and, accordingly, gives rise to a delay of the propagating pulse.

In our experiment, the steep dispersion is induced by ${ }^{57} \mathrm{Fe}$ nuclei, which are embedded in a nm-sized $\mathrm{x}$-ray cavity. A SNXP is reflected off this cavity in grazing incidence, and the reflected light is subsequently detected, as shown in Fig. 1. In this process the spectral properties of the x-ray field are modified by the complex reflection coefficient $R(\omega)$. Noting that $R=\exp [i \arg (R)+\log (|R|)]$ and by comparison with the expressions above, one finds that in our setup the phase of the reflection coefficient $\arg (R)$ takes the role of the real part of the susceptibility $\operatorname{Re}(\chi)$, while the logarithm of the modulus $\log (|R|)$ is connected to the imaginary part $\operatorname{Im}(\chi)$ (see Supplemental Material [28] for details). Hence, the time domain properties of the SNXP can be controlled via the phase of the reflection coefficient.

In order to derive the time delay experienced by the pulse, we characterize the input SNXP by $E(\omega)$ in the frequency domain and by the Fourier transform $E_{1}(t) \propto$ $\int E(\omega) e^{-i \omega t} d \omega$ in the time domain. The cavity modifies the SNXP spectrum via its complex reflection coefficient $R(\omega)$, which, for instance, can be calculated with the quantum optical model introduced in Ref. [9] (for details see the Supplemental Material [28]). Assuming that the pulse $E(\omega)$ is spectrally narrow around its center frequency $\omega_{0}$ compared to the superradiantly broadened cavity reflectance [13], we can approximate $R(\omega) \approx R\left(\omega_{0}\right) \exp \left[i\left(\omega-\omega_{0}\right) \tau\right]$, where

$$
\tau=\frac{\partial}{\partial \omega} \arg \left[R\left(\omega_{0}\right)\right]
$$

such that the temporal response of the setup is given by

$$
\begin{aligned}
E_{2}(t) & \propto \int E(\omega) R(\omega) e^{-i \omega t} d \omega \\
& \approx R\left(\omega_{0}\right) e^{-i \omega_{0} \tau} \int E(\omega) e^{-i \omega(t-\tau)} d \omega \\
& \propto E_{1}(t-\tau) .
\end{aligned}
$$

We thus find that the SNXP is delayed by the time $\tau$ due to the cavity dispersion, as it is well known from cavities and waveguides in the visible regime [32,33]. The narrow linewidth of Mössbauer transitions gives rise to considerable phase changes in a small frequency range around the resonance, consequently a steep dispersion and, hence, enables large time delays. To avoid distortions of the pulse shape, the dispersion should be linear over the complete width of the SNXP, which in our case is achieved by letting the pulse interact with the superradiantly broadened ensemble of nuclei in the cavity. This way, the dispersion is not as

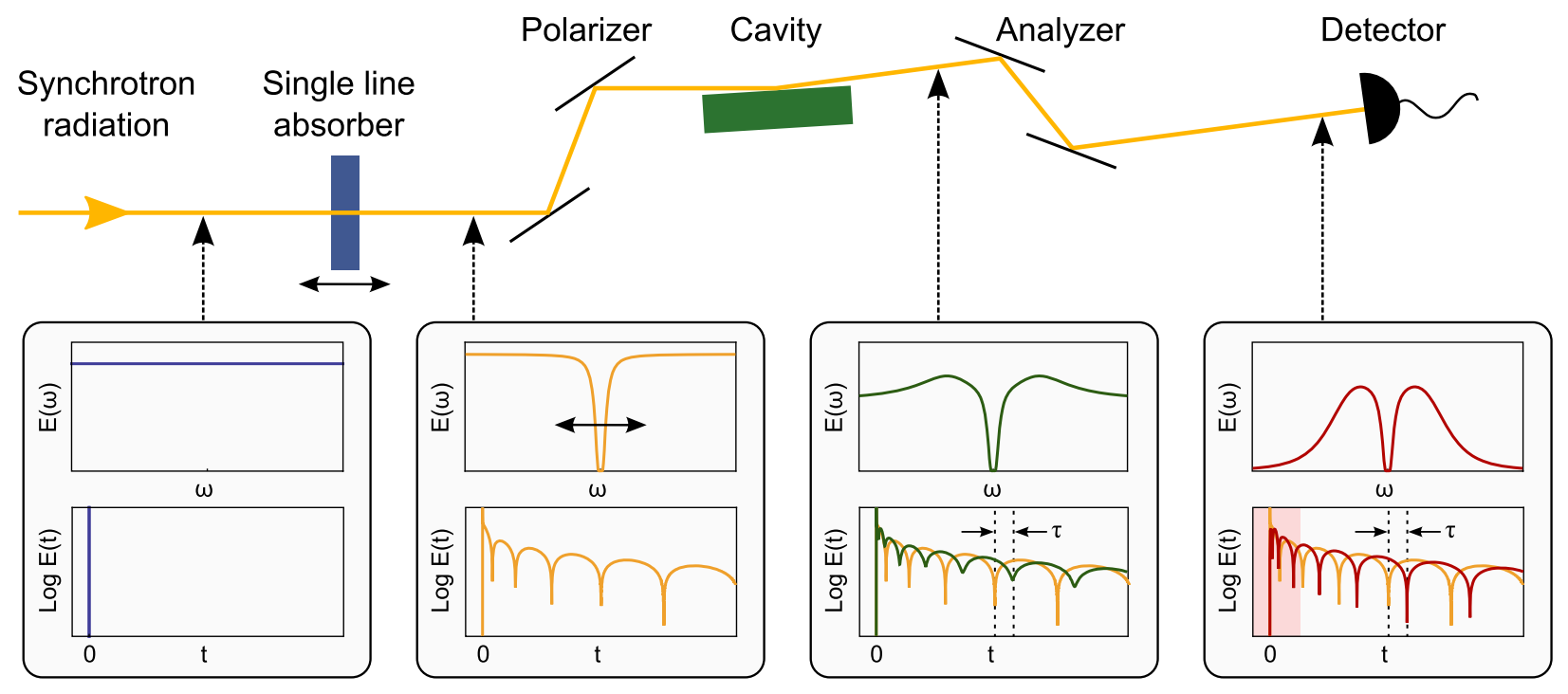

FIG. 1 (color online). Concept of the experiment: A single line absorber (SLA), mounted on a Doppler drive, imprints an absorption band on the broadband SR pulse, effectively generating a spectrally narrow X-ray pulse (SNXP) plus an unscattered constant contribution. Next, the $\mathrm{x}$ ray is reflected off of a thin-film cavity containing near-resonant nuclei. The nuclei imprint a spectral dispersion onto the pulse, which results in a temporal delay. Detuning the SNXP frequency via Doppler shifts allows one to tune the pulse delay. The polarimeter around the cavity blocks the constant offset of nonresonant background photons such that neither mechanical chopper nor a high-resolution monochromator for the SR are required. The residual signal of the unscattered constant contribution, which interacted with the cavity and passed the polarimeter is filtered out by variable a posteriori time gating (red shaded area). The delay of the SNXP is most clearly visible in comparing the beating minima in the time domain. The lower panels depict the frequency- and timeresolved amplitude of the field, and the two curves in the bottom right panels show the pulse with and without the induced delay $\tau$. In the experiment, the SLA was placed behind the analyzer (see main text). 
steep as for a single resonance, but extends over the whole SNXP. The group delay $\tau$ can in principle be tuned via a Doppler shift induced by moving the cavity, such that the cavity spectrum $R(\omega)$ is detuned with respect to the pulse spectrum $E(\omega)$, or by exploiting the dependence of $R(\omega)$ on the magnetization or polarization properties of the iron nuclei and the $\mathrm{x}$ rays, respectively.

SNXP generation.-The desired group velocity control and subsequent applications require a SNXP as input field, such that the steep linear nuclear dispersion covers the complete SNXP spectrum. In the X-ray regime, narrowband radiation is provided by Mössbauer radioactive sources [17], but these offer pulsed operation only in the single-photon regime. An alternative is provided by synchrotron radiation (SR) sources, where nuclear resonant scattering (NRS) is an established method [34]. SR experiments rely on broadband excitation of nuclear levels and subsequent detection of the delayed nuclear decay signal. Compared to Mössbauer radioactive sources, state-of-theart SR sources offer an average brilliance of photons in resonance with the nuclei enhanced by several orders of magnitude. Nevertheless, they are still restricted to less than one resonant photon per pulse on average. However, they provide a route towards the nonlinear regime, since future source developments could increase the number of resonant photons per pulse while preserving the pulsed nature of the source. Similarly, cavity-based setups will assist one in reaching this limit with photon fluences that are well below those required in free space. In addition, $\mathrm{x}$-ray free electron lasers (XFEL) already operate in a suitable frequency regime [35] and could in the future provide multiple resonant photons per shot in a pulsed operation for nuclear quantum optics. This prompts the question for SNXP generation from SR or XFEL sources. However, narrowband filtering of a single line from broadband radiation with sufficient rejection ratio is challenging since the beam has a bandwidth orders of magnitude larger than the nuclear resonance. One approach in this direction has been recently successfully demonstrated [36-38]. In this case, a narrowband, pure nuclear reflection from a ${ }^{57} \mathrm{FeBO}_{3}$ crystal is employed to suppress the enormous fraction of nonresonant photons in the incident beam. Another approach relies on a high-speed mechanical chopper [39]. In this method, the initial broadband SR pulse interacts with a single-line resonant energy analyzer (SLA), which acts equivalently to a spectrally narrow monochromator, but also contains the unscattered constant contribution. The chopper is operated such that it blocks this temporally short broadband signal, but lets the delayed signal pass. As a result, the spectral dip induced by the SLA is converted into a SNXP.

In our experiment, we generate the SNXP using a related method based on polarization filtering for the suppression of the background photons. In our setup, shown in Fig. 1, the initial broadband SR pulse interacts with the SLA, again resulting in a SNXP plus a broadband signal.
We eliminate this unwanted broadband contribution in two stages. First, the $\mathrm{x}$ rays are directed into a high purity $\mathrm{x}$-ray polarimeter consisting of a polarizer and analyzer in crossed setting [40-42], between which the cavity containing the nuclei is placed. Thus, only those photons arrive at the detector, whose polarization has been rotated by the interaction with the nuclei. Thereby, the nonresonant background and those photons which did not interact with the nuclei are eliminated. Because of the high purity of the polarimetry setup, neither a high-resolution monochromator for the incident SR pulse nor time gating of the detection are required in our setup. The detected signal becomes $R(t)=R_{\mathrm{SNXP}}(t)+R_{\mathrm{NI}}(t)$, where $R_{\mathrm{SNXP}}(t)$ is the desired delayed SNXP. $R_{\mathrm{NI}}(t)$ corresponds to photons which interacted with the nuclei in the cavity, but did not interact with the SLA. On average, $R_{\mathrm{SNXP}}(t)$ dominates over $R_{\mathrm{NI}}(t)$ for times $t \gtrsim 50 \mathrm{~ns}$. Therefore, the desired spectrally narrow delayed part $R_{\mathrm{SNXP}}(t)$ can be separated from $R_{\mathrm{NI}}(t)$ by time gating. Since the polarimeter enables us to record $R(t)$ for all times, this time gating can be applied and optimized throughout the data analysis. The connection of our setup to the scheme involving a mechanical chopper [39] is discussed in more detail in the Supplemental Material [28].

Experiment.-We performed the experiment at the nuclear resonance beam line (ID18) [43] of the European Synchrotron Radiation Source (ESRF, Grenoble). Compared to the setup sketched in Fig. 1, the order of the cavity and the SLA are reversed. This is possible, since all responses are linear. The $\mathrm{x}$-ray cavity consists of a $\mathrm{Pd}(2 \mathrm{~nm}) / \mathrm{C}(20 \mathrm{~nm}) /{ }^{57} \mathrm{Fe}(3 \mathrm{~nm}) / \mathrm{C}(21 \mathrm{~nm}) / \mathrm{Pd}(10 \mathrm{~nm}) /$ $\mathrm{Si}$ layer system which is probed in grazing incidence such that the fundamental guided cavity mode is resonantly excited [9]. The high purity x-ray polarimeter is described in more detail in [42]. A magnetic field is applied along the beam propagation direction, defining the quantization axis for the magnetic hyperfine splitting in the ${ }^{57} \mathrm{Fe}$ layer. In this setting, vacuum-mediated couplings between the different hyperfine levels arise, which lead to steep linear dispersion as in EIT systems $[9,16]$, such that large time delays $\tau$ are expected. Note that in contrast to previous experiments focusing on the measurement of the absorption spectra $[15,16]$, here, full transparency of the medium on resonance is not desirable, as it would correspond to zero intensity in reflection, prohibiting a detection of the propagated pulse. Therefore, the cavity system is chosen such that steep dispersion is obtained while maintaining sufficient intensity in reflection direction to enable the pulse detection. The SLA is formed by a $10 \mu \mathrm{m}$ thick stainless steel foil $\left({ }^{57} \mathrm{Fe}_{55} \mathrm{Cr}_{25} \mathrm{Ni}_{20}\right)$ with ${ }^{57} \mathrm{Fe}$ for the SNXP generation. This generates a SNXP with a length of $\approx 10 \mathrm{~ns}$. The SLA was mounted on a Doppler drive, such that pulses with different central frequencies $\omega_{\mathrm{SNXP}}=\omega_{0}+\Delta_{D}$ could be generated.

Because of the narrow nuclear linewidth, the SNXP consists, on average, of less than one photon. Triggering 

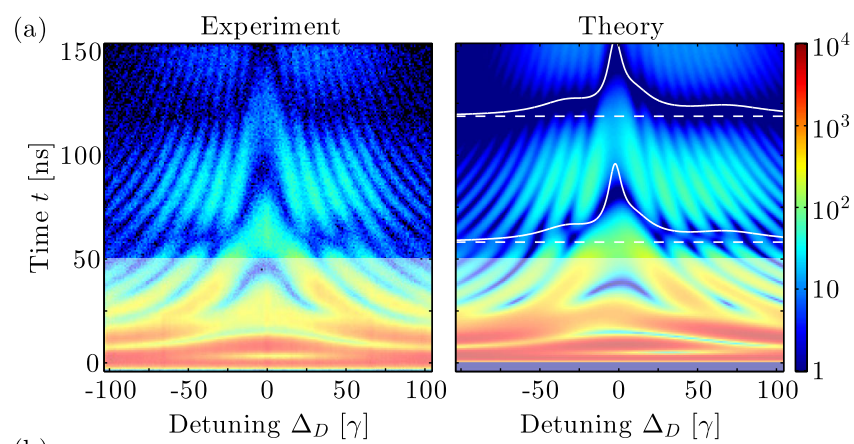

(b)

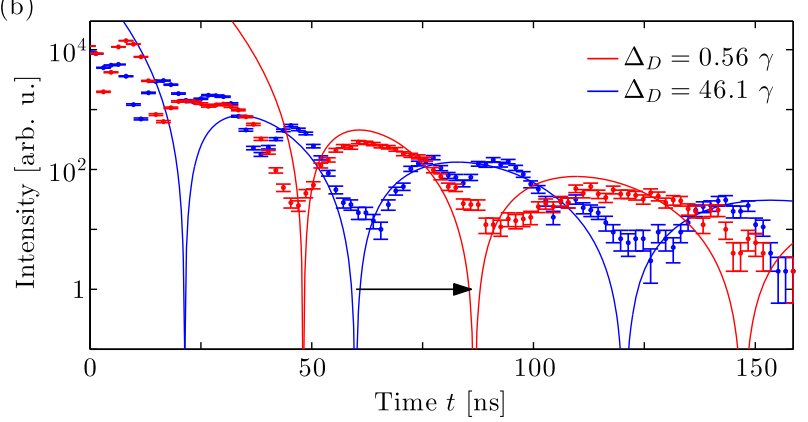

FIG. 2 (color online). (a) Photon counts as a function of time and Doppler detuning of the single line analyzer. Black pixels mark zero counts in the experiment. White dashed lines indicate theoretical predictions for beating minima positions without pulse delay. Solid white curves show corresponding predictions including the pulse delay. The additional oscillatory structure superimposing the data is due to photons $R_{\mathrm{NI}}(t)$. The bleached area $t \leq 50$ ns contains mostly data from $R_{\mathrm{NI}}(t)$ and is excluded from the data analysis, whereas times $t>50 \mathrm{~ns}$ contain mostly the desired $R_{\mathrm{SNXP}}(t)$. (b) Sections through (a) at constant detuning $\Delta_{D}$. Close to resonance $\Delta_{D} \approx 0$, the temporal response is clearly shifted compared to the off-resonant case. For example, the minimum at $t \approx 60 \mathrm{~ns}$ is shifted to later times as indicated by the arrow. Solid lines are theoretical predictions for the pulse part $R_{\mathrm{SNXP}}$ only, which is expected to deviate from the experimental data at initial times due to the omission of $R_{\mathrm{NI}}(t)$.

data acquisition on the detection of a photon at the detector thus essentially leads to postselection of single photon SNXP. In the experiment, we registered the photon time of arrival together with the Doppler drive velocity for each signal photon separately. This enables us to analyze the intensity of the light registered by the detector as a function of the pulse center frequency and time, as shown in Fig. 2. Clearly, the time spectra of near-resonant pulses $\left(\Delta_{D} \approx 0\right)$ are delayed compared to those of the off-resonant pulses, which can be seen, e.g., from the shift of a beating minimum at $t \approx 60 \mathrm{~ns}$ to later times; see arrow in Fig. 2(b). This figure also shows that the SNXP structure remains essentially undistorted. The agreement between experimental data and theoretical predictions is very good. From the theoretical analysis, we could also identify the additional oscillatory structures superimposing the simple temporal shift of the registered intensity by $\tau$ predicted in Eq. (2) as arising from residuals of $R_{\mathrm{NI}}(t)$ in the data.

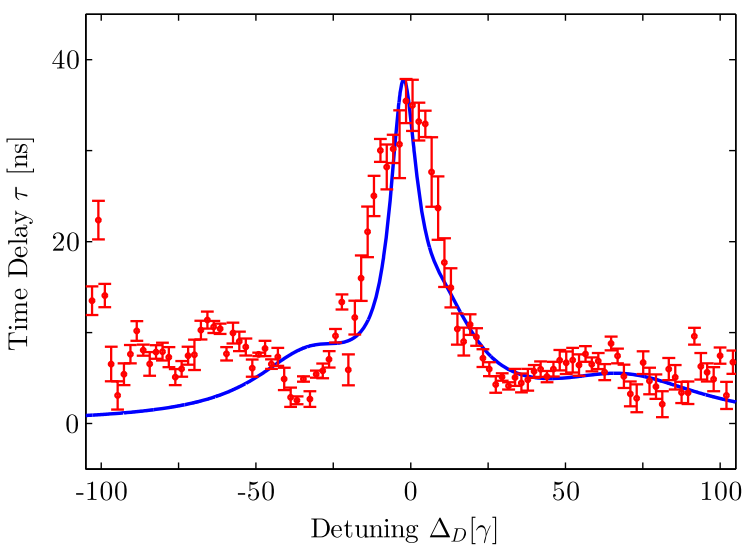

FIG. 3 (color online). Time delay for the SNXP as a function of the detuning $\Delta_{D}$ between SNXP and the nuclear resonance. Red dots show the delay extracted from the experimental data. The blue solid curve shows the corresponding theoretical prediction. Error bars are described in the Supplemental Material [28].

From the experimental data shown in Fig. 2, we extracted the time delay $\tau$ of the x-ray pulses by fitting the analytical response function with variable $\tau$ to the data (Details on the employed fit method are provided in the Supplemental Material [28]). The result is shown in Fig. 3. As expected, around the cavity resonance where the nuclear susceptibility exhibits a steep positive linear dispersion, substantial pulse delays up to $35 \mathrm{~ns}$ are observed. Away from the nuclear resonance, the delay reduces until it becomes zero off-resonance.

We have thus demonstrated group velocity control for spectrally narrow hard $\mathrm{x}$-ray pulses, yielding controllable pulse delays of up to $35 \mathrm{~ns}$ via subluminal light propagation. The subluminal light propagation was realized by tailoring a suitable steep linear dispersion in Mössbauer nuclei embedded in an x-ray cavity. Our theoretical analysis agrees well with the experimental results. From numerical finite-difference time-domain simulations of the x-ray pulse dynamics, we determined an upper bound for the propagation length of the pulse inside the cavity of about $1 \mathrm{~mm}$, which translates into an upper bound for the reduced group velocity of the SNXP of $v_{\mathrm{gr}}<10^{-4} c$.

These results pave the way for a number of promising future directions. Our theory predicts that a suitable modification of sample magnetization and $\mathrm{x}$-ray polarization offers means to tune the SNXP group velocity and thus the time delay, even to superluminal light propagation $[32,44]$. Also a storage of $\mathrm{x}$-ray photons could be envisioned. This way, slow light, EIT, and related phenomena in the future could enable the coherence-based enhancement of nonlinear interaction between $\mathrm{x}$ rays and nuclei [1]. Next to this primary goal, our setup in turn could also be used to measure the phase of the response of an unknown sample, since the observed time delay is directly related to the phase of the optical response of the cavity-nuclei system. 
K.P.H. acknowledges funding by the German National Academic Foundation and G. G. P. funding by Deutsche Forschungsgemeinschaft in the framework of CRC TR18.

[1] M. Fleischhauer, A. Imamoglu, and J. P. Marangos, Rev. Mod. Phys. 77, 633 (2005).

[2] Focus issue on slow light, Nat. Photonics 2, 447 (2008).

[3] Cavity Quantum Electrodynamics, Advances in Atomic, Molecular and Optical Physics, edited by P. R. Berman (Academic Press, New York, 1994).

[4] O. Kocharovskaya, R. Kolesov, and Y. Rostovtsev, Phys. Rev. Lett. 82, 3593 (1999).

[5] A. Pálffy, C. H. Keitel, and J. Evers, Phys. Rev. Lett. 103, 017401 (2009).

[6] T. J. Bürvenich, J. Evers, and C. H. Keitel, Phys. Rev. Lett. 96, 142501 (2006).

[7] N. ten Brinke, R. Schützhold, and D. Habs, Phys. Rev. A 87, 053814 (2013).

[8] W.-T. Liao, A. Pálffy, and C. H. Keitel, Phys. Rev. Lett. 109, 197403 (2012).

[9] K. P. Heeg and J. Evers, Phys. Rev. A 88, 043828 (2013).

[10] Y. V. Shvyd'ko, T. Hertrich, U. van Bürck, E. Gerdau, O. Leupold, J. Metge, H. D. Rüter, S. Schwendy, G. V. Smirnov, W. Potzel, and P. Schindelmann, Phys. Rev. Lett. 77, 3232 (1996).

[11] R. Coussement, Y. Rostovtsev, J. Odeurs, G. Neyens, H. Muramatsu, S. Gheysen, R. Callens, K. Vyvey, G. Kozyreff, P. Mandel, R. Shakhmuratov, and O. Kocharovskaya, Phys. Rev. Lett. 89, 107601 (2002).

[12] R. N. Shakhmuratov, F. Vagizov, J. Odeurs, and O. Kocharovskaya, Phys. Rev. A 80, 063805 (2009).

[13] R. Röhlsberger, K. Schlage, B. Sahoo, S. Couet, and R. Rüffer, Science 328, 1248 (2010).

[14] R. N. Shakhmuratov, F. Vagizov, and O. Kocharovskaya, Phys. Rev. A 84, 043820 (2011).

[15] R. Röhlsberger, H.-C. Wille, K. Schlage, and B. Sahoo, Nature (London) 482, 199 (2012).

[16] K. P. Heeg, H.-C. Wille, K. Schlage, T. Guryeva, D. Schumacher, I. Uschmann, K. S. Schulze, B. Marx, T. Kämpfer, G. G. Paulus, R. Röhlsberger, and J. Evers, Phys. Rev. Lett. 111, 073601 (2013).

[17] F. Vagizov, V. Antonov, Y. V. Radeonychev, R. N. Shakhmuratov, and O. Kocharovskaya, Nature (London) 508, 80 (2014).

[18] Nonlinear Optics, Quantum Optics, and Ultrafast Phenomena with X-Rays: Physics with X-Ray Free-Electron Lasers, edited by B. W. Adams (Springer, Heidelberg, 2003).

[19] B. W. Adams, C. Buth, S. M. Cavaletto, J. Evers, Z. Harman, C. H. Keitel, A. Pálffy, A. Picon, R. Röhlsberger, Y. Rostovtsev, and K. Tamasaku, J. Mod. Opt. 60, 2 (2013).

[20] K. P. Heeg, C. Ott, D. Schumacher, H.-C. Wille, R. Röhlsberger, T. Pfeifer, and J. Evers, Phys. Rev. Lett. 114, 207401 (2015).

[21] L. V. Hau, S. E. Harris, Z. Dutton, and C. H. Behroozi, Nature (London) 397, 594 (1999).
[22] M. M. Kash, V. A. Sautenkov, A. S. Zibrov, L. Hollberg, G. R. Welch, M. D. Lukin, Y. Rostovtsev, E. S. Fry, and M. O. Scully, Phys. Rev. Lett. 82, 5229 (1999).

[23] D. Budker, D. F. Kimball, S. M. Rochester, and V. V. Yashchuk, Phys. Rev. Lett. 83, 1767 (1999).

[24] M. Mücke, E. Figueroa, J. Bochmann, C. Hahn, K. Murr, S. Ritter, C. J. Villas-Boas, and G. Rempe, Nature (London) 465, 755 (2010).

[25] M. Albert, A. Dantan, and M. Drewsen, Nat. Photonics 5, 633 (2011).

[26] J. Odeurs, G. Hoy, Y. Rostovtsev, and R. Shakhmuratov, Laser Photonics Rev. 4, 1 (2010).

[27] S. E. Harris, J. E. Field, and A. Kasapi, Phys. Rev. A 46, R29 (1992).

[28] See Supplemental Material at http://link.aps.org/ supplemental/10.1103/PhysRevLett.114.203601 for the relation between $R$ and $\chi$, the theoretical model, details on the SNXP generation and on the method of curve fitting, which includes Refs. [29-31].

[29] Y. Kagan, A. M. Afanas'ev, and V. G. Kohn, J. Phys. C 12, 615 (1979).

[30] U. van Bürck, Hyperfine Interact. 123/124, 483 (1999).

[31] J. Hannon and G. Trammell, Hyperfine Interact. 123/124, 127 (1999).

[32] S. Longhi, M. Marano, P. Laporta, M. Belmonte, and P. Crespi, Phys. Rev. E 65, 045602 (2002).

[33] J. E. Heebner and R. W. Boyd, J. Mod. Opt. 49, 2629 (2002).

[34] R. Röhlsberger, in Nuclear Condensed Matter Physics with Synchrotron Radiation, Springer Tracts in Modern Physics (Springer Berlin Heidelberg, 2005), Vol. 208, p. 67.

[35] T. Ishikawa, H. Aoyagi, T. Asaka, Y. Asano et al., Nat. Photonics 6, 540 (2012).

[36] G. V. Smirnov, U. van Bürck, A. I. Chumakov, A. Q. R. Baron, and R. Rüffer, Phys. Rev. B 55, 5811 (1997).

[37] T. Mitsui, M. Seto, R. Masuda, K. Kiriyama, and Y. Kobayashi, Jpn. J. Appl. Phys. 46, L703 (2007).

[38] V. Potapkin, A. I. Chumakov, G. V. Smirnov, J.-P. Celse, R. Rüffer, C. McCammon, and L. Dubrovinsky, J. Synchrotron Radiat. 19, 559 (2012).

[39] T. S. Toellner, E. E. Alp, T. Graber, R. W. Henning, S. D. Shastri, G. Shenoy, and W. Sturhahn, J. Synchrotron Radiat. 18, 183 (2011).

[40] T. S. Toellner, E. E. Alp, W. Sturhahn, T. M. Mooney, X. Zhang, M. Ando, Y. Yoda, and S. Kikuta, Appl. Phys. Lett. 67, 1993 (1995).

[41] R. Röhlsberger, E. Gerdau, R. Rüffer, W. Sturhahn, T. Toellner, A. Chumakov, and E. Alp, Nucl. Instrum. Methods Phys. Res., Sect. A 394, 251 (1997).

[42] B. Marx, I. Uschmann, S. Höfer, R. Lötzsch, O. Wehrhan, E. Förster, M. Kaluza, T. Stöhlker, H. Gies, C. Detlefs, T. Roth, J. Härtwig, and G. Paulus, Opt. Commun. 284, 915 (2011).

[43] R. Rüffer and A. I. Chumakov, Hyperfine Interact. 97-98, 589 (1996).

[44] S. Chu and S. Wong, Phys. Rev. Lett. 48, 738 (1982). 